\title{
ON CLOSURE CONDITIONS OF DENSE IRREDUCIBLE SUBALGEBRAS OF COMPACT ELEMENTARY OPERATORS
}

\author{
W. KANGOGO*, N. B. OKELO, O. ONGATI \\ Department of Pure and Applied Mathematics, \\ Jaramogi Oginga Odinga University of Science and Technology, \\ Box 210-40601, Bondo-Kenya \\ *Corresponding author: kangogowll@gmail.com \\ Received Jan. 15, 2021
}

\begin{abstract}
In this paper, we give some properties of spectrally bounded compact elementary operators on dense irreducible subalgebras and determine the closure conditions for the centre of spectrally bounded compact elementary operators on dense irreducible subalgebras. 2010 Mathematics Subject Classification. 47B47; 47A30.
\end{abstract}

Key words and phrases. centre; elementary operators; Spectrally bounded.

\section{InTRODUCTION}

The centre of a $C^{*}$-algebra $\mathcal{A}$ contains information about properties of operators defined on $\mathcal{A}$ which are compatible with ideals of $\mathcal{A}$ such as derivations, automorphisms, elementary operators, among others [1]. The study of spectral boundedness of elementary operators was introduced by Mathieu [2] in which the results were motivated by their connections to the noncommutative Singer-Wermer conjuncture and Kaplansky's problem of invertibility-preserving operators. Lin and Mathieu [3] proved that every unital bounded linear mapping from a purely infinite $C^{*}$-algebra of real rank zero into a unital Banach algebra which preserves elements of square zero was a Jordan homomorphism. The authors in [4] studied the norm property of a two-sided multiplication elementary operator and showed that $\left\|M_{A, B}(X)\right\|=\|A\|\|B\|$. Furthermore, a number of properties of elementary operators have been studied in, [5], [6], [7] and [8]. Molnar and Semrl [9] characterized surjective maps between standard operator 
algebras having a certain multiplicativity-like appearing in the abstract definition of elementary operators of length one. Furthermore, the structure [10], Theorem 3.5, $S=M_{A, B}+M_{C, D} \in \mathcal{C}(\mathcal{A})$ has been studied and found to be spectrally bounded if and only if $B A+D C \in Z(\mathcal{A})$. However if the centre of $\mathcal{A}$ is enlarged then it is not well behaved in terms of spectral boundedness. Also, Mathieu possed the question in [11],Theorem 2.4 that "Is it true that commutator $[X, \delta(X)]$ belongs to the $Z(\mathcal{A})$ for every $X \in \mathcal{A}$ imply that the inner derivation is spectrally bounded?" Thus, there are alot of researches in attempt to answer the above questions. In addition, Young [12] provided criteria when an elementary operator is spectrally bounded or spectrally isometric in attempt to answer why elementary operators still resist a smooth general theory. Furthermore, Mathieu and Young [13] studied spectrally isometric elementary operators and outlined criteria for unital elementary operators on unital semi-simple Banach algebras to be spectral isometries since there is no relationship between boundedness and spectral boundedness of a linear mapping in general. Therefore, whenever we have a spectrally bounded operator on algebra does not imply that it is spectrally bounded in general. Hence, this prompts that there is still room to study structural properties of spectrally bounded compact elementary operators on dense irreducible subalgebras. In addition, Rennison [14] gave a number of conditions related to centrality of Banach algebras which include nonunital algebras, analytic functions of quasi central elements and algebras having all quasi central elements which are central. Sarsour and As'ad [15] studied the conditions under which quasi-centrality is preserved under the mapping and established the relationship between centrality of Banach algebras and centrality of its closed subalgebras. As'ad [16] studied the centrality of a non-unital complex Banach algebra and generalized some results related to centrality in a non-unital Banach algebra. Moreover, in [16] the author showed that under certain conditions that the set of all $\rho$-quasi central elements of non-unital complex Banach algebra is a subset of the set of all $\rho$-quasi central elements of $\mathcal{A}^{*}$ where $\mathcal{A}^{*}$ is the unitazation of $\mathcal{A}$. Furthermore, As'ad [16] showed that under the same conditions, the $\rho$-quasi-centre, $\sigma$-quasi-centre and quasi-centre of $\mathcal{A}$ can be preserved under the quotient mapping and in [17] As'ad gave the extended centre, extended quasi-centre, extended $\sigma$-quasi centre and extended $\rho$-quasi centre properties of complex Banach algebra. Duggal [18] studied the range closure of an elementary operator. Let $B(H)$ denote the algebra of operators on Hilbert space $H$. Let $\Delta_{A, B} \in B(B(H))$ and $E \in B(B(H))$ denote the elementary operators $\Delta_{A B}(X)=A X B-X$ and $E(X)=A X B-C X D$. Duggal [18] showed that if $A, B$ are 
contractions then $B(H)=\Delta_{A, B}^{-1}(0) \oplus \Delta_{A, B}(B(H))$ if and only if $\Delta_{A, B}^{n}(B(H))$ is closed for some integer $n \geq 1$. Moreover, the authors in [14] and [19] outlined several properties of the centre and quasi-centre of the semi-simple Banach algebras such as any $C^{*}$-algebra, the algebra $\mathrm{B}(\mathrm{X})$ of all bounded linear operators on any Banach space $X$ or group algebra $L^{1}(G)$ of any compact group $G$. The following objectives guided the study. Therefore, in this paper, we determine the closure conditions for the centre of spectrally bounded compact elementary operators on dense irreducible subalgebras.

\section{Preliminaries}

In this section, we outline preliminary concepts which are useful in the sequel. Let $\mathcal{A}$ be a Banach algebra and $\mathcal{A}_{\mathcal{D I R}}$ be a dense irreducible $C^{*}$-subalgebra of $\mathcal{A}$. We denote the algebra of all compact elementary operators on $\mathcal{A}$ by $\mathcal{C}(\mathcal{A})$ and algebra of all spectrally bounded compact elementary operators on $\mathcal{A}_{\mathcal{D I R}}$ by $\mathcal{C}_{\mathcal{S B D}}\left(\mathcal{A}_{\mathcal{D I R}}\right)$. For an elementary operator $T_{A_{i}, B_{i}}(X)=A_{i} X B_{i} \in$ $\mathcal{C}_{\mathcal{S B D}}\left(A_{D I R}\right)$, we define the linear span of $A, B$ and $B A$ by $\mathbf{A}\left(T_{A_{i}, B_{i}}\right)=\operatorname{span}\left\{A_{i}, \ldots, A_{n}\right\}$, $\mathbf{B}\left(T_{A_{i}, B_{i}}\right)=\operatorname{span}\left\{B_{i}, \ldots, B_{n}\right\}$ and $\mathbf{C}\left(T_{A_{i}, B_{i}}\right)=\operatorname{span}\left\{B_{i} A_{j} ; 1 \leq i, j \leq n\right\}$ respectively.

Definition 2.1. ( [7], Section 2) Consider a $C^{*}$-algebra $\mathcal{A}$ and let $T: \mathcal{A} \rightarrow \mathcal{A}$. The operator $T$ is called an elementary operator if it has the following representation: $T_{A_{i}, B_{i}}(X)=\sum_{i=1}^{n} A_{i} X B_{i} \forall X \in \mathcal{A}$ and $A_{i}, B_{i}$ are fixed in $\mathcal{A}$ or $\mathcal{M}(\mathcal{A})$ is multiplier algebra of $\mathcal{A}$. For $A, B \in B(H)$, we define particular elementary operators

(i). the left multiplication operator $L_{A}: B(H) \rightarrow B(H)$ by $L_{A}(X)=A X, \forall X \in B(H)$.

(ii). the right multiplication operator $R_{B}: B(H) \rightarrow B(H)$ by $R_{B}(X)=X B, \forall X \in B(H)$.

(iii). the generalized derivation (implemented by $A, B)$ by $\delta_{A, B}(X)=L_{A}-R_{B}, \forall X \in B(H)$.

(iv). the basic elementary operator (implemented by $A, B)$ by $M_{A, B}(X)=A X B, \forall X \in B(H)$.

(v). The Jordan elementary operator (implemented by $A, B$ ) by $\mathfrak{U} A, B(X)=A X B+B X A, \forall X \in$ $B(H)$.

Definition 2.2. ( [20], Definition 1.3) The centre of a Banach algebra $\mathcal{A}$ is defined by $\mathcal{Z}(\mathcal{A})=\{A \in$ $\mathcal{A}: A X=X A, \forall X \in \mathcal{A}\}$.

Definition 2.3. The quasi-centre $W\left[\mathcal{C}_{\mathcal{S B D}}(\Omega)\right]$ of $\mathcal{C}_{\mathcal{S B D}}\left(\mathcal{A}_{\mathcal{D I R}}\right)$ is the set of all quasi-central of $A \in$ $\mathcal{C}_{\mathcal{S B D}}\left(\mathcal{A}_{\mathcal{D I R}}\right)$ defined by $W\left[\mathcal{C}_{\mathcal{S B D}}(\Omega)\right]=\bigcup_{\omega \in \mathbb{K}}\{\|X(\lambda-A)\| \leq \omega\|(\lambda-A) X\|$, for every $X \in$ $\mathcal{C}_{\mathcal{S B D}}\left(\mathcal{A}_{\mathcal{D I R}}\right)$ and $\left.\omega \in \mathbb{K}\right\}$. 
Definition 2.4. Let $\mathcal{C}_{\mathcal{S B D}}\left(\mathcal{A}_{\mathcal{D I R}}\right)$ be an irreducible $C^{*}$-subalgebra and $\mathcal{A}_{\mathcal{S B D}} \subseteq \mathcal{C}_{\mathcal{S B D}}\left(\mathcal{A}_{\mathcal{D I R}}\right)$. Then interior $\mathcal{A}_{\mathcal{S B D}}$ is denoted $\operatorname{Int}\left(\mathcal{A}_{\mathcal{S B D}}\right)=\left\{T \in \mathcal{A}_{\mathcal{S B D}} \mid D=\{\|T\| \leq 1\} \subset \mathcal{A}_{\mathcal{S B D}}\right\}$, where $D$ is unit disk and the closure of $\mathcal{A}_{\mathcal{S B D}}$ denoted by $\overline{\mathcal{A}_{\mathcal{S B D}}}$ is set defined by $\overline{\mathcal{A}_{\mathcal{S B D}}}=\left\{T \in \mathcal{C}_{\mathcal{S B D}}\left(\mathcal{A}_{\mathcal{D I R}}\right) \mid \lim _{n \rightarrow \infty} T_{n}=\right.$ $\left.T, \forall T_{n} \in \mathcal{A}_{\mathcal{S B D}}\right\}$.

Definition 2.5. ( [21], Definition 3) If $\mathcal{A}$ is any $C^{*}$-algebra and $\Phi: \mathcal{A} \rightarrow B(H)$ is a representation then $\Phi$ is irreducible if $\Phi(\mathcal{A})$ is an irreducible subalgebra of $B(H)$.

Definition 2.6. ( [21], Definition 4) Let $\mathcal{A}$ and $\mathcal{B}$ be complex Banach algebras. A linear mapping $T: \mathcal{A} \rightarrow \mathcal{B}$ is called spectrally bounded if there exists a constant $M \geq 0$ such that $r(T x) \leq M r(x)$ and spectrally infinitesimal if $r(T(x))=0$, for all $x \in \mathcal{A}$. If $r(T(x))=r(x)$, for all $x \in \mathcal{A}$ we say that $T$ is a spectral isometry. If $r(x)=0$, then $x$ is called quasi-nilpotent.

Remark 2.1. If $\left(L_{A}-R_{A}\right) \mathcal{A}_{\mathcal{D I R}} \subsetneq \operatorname{Rad}\left(\mathcal{A}_{\mathcal{D I R}}\right)$ that is $A$ is not central modulo $\operatorname{Rad}\left(\mathcal{A}_{\mathcal{D I R}}\right)$ then $R_{A}$ and hence $L_{A}$ cannot be spectrally bounded.

\section{MAIN RESULTS}

In this section, we first outline the centre properties of dense irreducible subalgebras of compact elementary operators that are spectrally bounded. See [21] for more details on the centre properties of dense irreducible subalgebras.

Proposition 3.1. Let $\mathcal{A}_{\mathcal{D I R}}, \mathcal{B}_{\mathcal{D I R}}, \mathcal{C}_{\mathcal{D I R}}$ be dense irreducible subalgebras and $S_{A_{i}, B_{i}}, T_{A_{i}, B_{i}}, T_{A_{i}, B_{i}} \in$ $\mathcal{C}_{\mathcal{S B D}}\left(\mathcal{A}_{\mathcal{D I R}}\right)$ such that $S_{A_{i}, B_{i}}: \mathcal{A}_{\mathcal{D I R}} \rightarrow \mathcal{B}_{\mathcal{D I R}}, T_{A_{i}, B_{i}}: \mathcal{A}_{\mathcal{D I R}} \rightarrow \mathcal{B}_{\mathcal{D I R}}$ and $R_{A_{i}, B_{i}}: \mathcal{B}_{\mathcal{D I R}} \rightarrow \mathcal{C}_{\mathcal{D I R}}$ then the following conditions hold:

(i). $\left\|T_{A_{i}, B_{i}}\right\|_{\sigma}=\sup \left\{r\left(T_{A_{i}, B_{i}} X\right) \mid X \in \mathcal{A}_{\mathcal{D I R}}, r(X) \leq 1\right\}=\sup \left\{r\left(T_{A_{i}, B_{i}} X\right)\right.$

$\left.\mid X \in \mathcal{A}_{\mathcal{D I R}}, r(X)=1\right\}$.

(ii). $\left\|\lambda T_{A_{i}, B_{i}}\right\|_{\sigma}=|\lambda|\left\|T_{A_{i}, B_{i}} \mid\right\| \forall \lambda \in \mathbb{C}$.

(iii). $\left\|R_{A_{i}, B_{i}} T_{A_{i}, B_{i}}\right\|_{\sigma} \leq\left\|R_{A_{i}, B_{i}}\right\|_{\sigma}\left\|T_{A_{i}, B_{i}}\right\|_{\sigma}$

(iv). $\left\|S_{A_{i}, B_{i}}+T_{A_{i}, B_{i}}\right\|_{\sigma} \leq\left\|S_{A_{i}, B_{i}}\right\|_{\sigma}+\left\|T_{A_{i}, B_{i}}\right\|_{\sigma}$ if $\mathcal{B}_{\mathcal{D I R}}$ is commutative.

Proof. (i). There exists a constant $M \geq 0$ such that $r\left(T_{A_{i}, B_{i}} X\right) \leq M r(X), X \in \mathcal{A}_{\mathcal{D I R}}$ holds. This means that $\left\|T_{A_{i}, B_{i}}\right\|_{\sigma}=\sup \left\{r\left(T_{A_{i}, B_{i}} X\right) \mid X \in \mathcal{A}_{\mathcal{D I R}}\right.$, $r(X) \leq 1\} \leq M$. Let $\alpha=\sup \left\{r\left(T_{A_{i}, B_{i}} X\right) \mid X \in \mathcal{A}_{\mathcal{D I R} \mathcal{R}}, r(X)=1\right\}$, when $\alpha=0$ then $\mathcal{B}_{\mathcal{D I R}}$ has non-zero elements. When $X$ is quasinilpotent in $\mathcal{A}_{\mathcal{D I R}}$ then $r\left(T_{A_{i}, B_{i}} X\right) \leq$ $\alpha r(X)$ holds since $T_{A_{i}, B_{i}}$ is spectrally bounded. Also when $X$ is not quasinilpotent 
in $\mathcal{A}_{\mathcal{D I R}}$ then $r\left(T_{A_{i}, B_{i}}\left(\frac{X}{r(X)}\right)\right) \leq \alpha$. Hence, $r\left(T_{A_{i}, B_{i}} X\right) \leq \alpha r(X), \forall X \in \mathcal{A}_{\mathcal{D I R}}$ which shows that $\alpha \geq\left\|T_{A_{i}, B_{i}}\right\|_{\sigma}$.

(ii).

$$
\begin{aligned}
\left\|\lambda T_{A_{i}, B_{i}}\right\|_{\sigma} & =\sup \left\{\lambda r\left(T_{A_{i}, B_{i}} X\right) \mid X \in \mathcal{A}_{\mathcal{D I R}}, r(X) \leq 1\right\} \\
& \leq|\lambda| \sup \left\{r\left(T_{A_{i}, B_{i}} X\right) \mid X \in \mathcal{A}_{\mathcal{D I R}}, r(X)=1\right\} \\
& =|\lambda|\left\|T_{A_{i}, B_{i}}\right\|_{\sigma} .
\end{aligned}
$$

(iii). We know that from (i). $r\left(T_{A_{i}, B_{i}} X\right) \leq\left\|T_{A_{i}, B_{i}}\right\|_{\sigma} r(X), X \in \mathcal{A}_{\mathcal{D I R}}$, hence $r\left(R_{A_{i}, B_{i}} T_{A_{i}, B_{i}} X\right) \leq$ $\left\|R_{A_{i}, B_{i}}\right\|_{\sigma} r\left(T_{A_{i}, B_{i}} X\right) \leq\left\|R_{A_{i}, B_{i}}\right\|_{\sigma}\left\|T_{A_{i}, B_{i}}\right\|_{\sigma}$ $r(X)$ for all $X \in \mathcal{A}_{\mathcal{D I R}}$. This shows that $\mathcal{B}_{\mathcal{D I R}}$ is commutative and the spectral radius is sub-additive.

(iv). When $\mathcal{B}_{\mathcal{D I R}}$ is commutative then for $X \in \mathcal{A}_{\mathcal{D I R}}$,

$$
\begin{aligned}
\left\|S_{A_{i}, B_{i}}+T_{A_{i}, B_{i}}\right\|_{\sigma}= & \sup \left\{r\left(S_{A_{i}, B_{i}} X\right)+r\left(T_{A_{i}, B_{i}} X\right) \mid, r(X) \leq 1\right\} \\
\leq & \sup \left\{r\left(S_{A_{i}, B_{i}} X\right) \mid X \in \mathcal{A}_{\mathcal{D I R}}, r(X) \leq 1\right\} \\
& +\sup \left\{r\left(T_{A_{i}, B_{i}} X\right) \mid X \in \mathcal{A}_{\mathcal{D I R}}, r(X) \leq 1\right\} \\
= & \left\|S_{A_{i}, B_{i}}\right\|_{\sigma}+\left\|T_{A_{i}, B_{i}}\right\|_{\sigma} .
\end{aligned}
$$

Proposition 3.2. Let $A, B \in \mathcal{C}_{\mathcal{S B D}}\left(\mathcal{A}_{\mathcal{D I R}}\right)$ and $P=B A$ such that the basic elementary operator $M_{A, B}(X)=A X B$ is homomorphism modulo $\operatorname{Rad}\left(\mathcal{C}_{\mathcal{S B D}}\left(\mathcal{A}_{\mathcal{D I R}}\right)\right)$, then $P$ is an idempotent in $Z\left[\mathcal{C}_{\mathcal{S B D}}\left(\mathcal{A}_{\mathcal{D I R}}\right)\right]$ and

$M_{A, B}(I-P) \mathcal{C}_{\mathcal{S B D}}\left(\mathcal{A}_{\mathcal{D I R}}\right) \subseteq \operatorname{Rad}\left(\mathcal{C}_{\mathcal{S B D}}\left(\mathcal{A}_{\mathcal{D I R}}\right)\right)$.

Proof. We show that $P=B A$ is an idempotent in $Z\left[\mathcal{C}_{\mathcal{S B D}}\left(\mathcal{A}_{\mathcal{D I R}}\right)\right]$. Suppose that there exists $v \in$ $\Omega$ such that the family $\{\Phi(B A) v, v\}$ is linearly independent for an irreducible representation $\Phi: \mathcal{C}_{\mathcal{S B D}}\left(\mathcal{A}_{\mathcal{D I R}}\right) \rightarrow \Omega$ on a Banach space $\Omega$. Then by Jacobson density theorem, then there exists $X, Y \in \mathcal{C}_{\mathcal{S B D}}\left(\mathcal{A}_{\mathcal{D I R}}\right)$ such that $\Phi(X B A) v=0, \Phi(X) v=v$ and $\Phi(Y B A) v=v$. Thus

$$
\begin{aligned}
\Phi\left(M_{A, B}(X Y) A\right) v & =\Phi(A X Y B A) v \\
& =\Phi(A X Y P) v \text { if } W=X Y \\
& =\Phi(A W P) v \\
& =v=\Phi(A) v
\end{aligned}
$$


and

$$
\begin{aligned}
\Phi\left(M_{A, B}(X) M_{A, B}(Y) A\right) v & =\Phi(A X B A Y B A) v \\
& =\Phi(A X P Y P) v \\
& =\Phi\left(A X Y P^{2}\right) v \\
& =P^{2} \Phi(A W) v \\
& =P^{2} .0=0
\end{aligned}
$$

which is a contradiction. Therefore, $\{\Phi(B A) v, v\}$ is linearly independent for all $v \in \Omega$ and $\Phi(B A) \in \mathbb{C}_{I}$ i.e $\Phi(B A)=\lambda I$. Suppose that $X, Y \in \mathcal{C}_{\mathcal{S B D}}\left(\mathcal{A}_{\mathcal{D I R}}\right)$ such that $\lambda \in\{0,1\}$, then

$$
\Phi(A X Y B)=\Phi(A X B A Y B) \Rightarrow \Phi((1-\lambda) A X Y B)=0 .
$$

Let $X=Y=1$ such that multiplying $B$ on left hand side of Equation 3.1 and multiplying $A$ on the right hand side of Equation 3.1, we have $\Phi((1-\lambda) B A X Y B A)=(1-\lambda) \lambda I .1 .1 . \lambda I=$ $(1-\lambda) \lambda^{2}=0$ which hold. Thus $P=B A$ is an idempotent $Z\left[\mathcal{C}_{\mathcal{S B D}}\left(\mathcal{A}_{\mathcal{D I R}}\right)\right]$. In addition, from Equation 3.1, $\Phi(A(I-P) X B)=\Phi((1-\lambda) A X B)=0$ for all $X \in \mathcal{C}_{\mathcal{S B D}}\left(\mathcal{A}_{\mathcal{D I R}}\right)$ which shows that $M_{A, B}(I-P) \mathcal{C}_{\mathcal{S B D}}\left(\mathcal{A}_{\mathcal{D I R}}\right) \subseteq \operatorname{Rad}\left(\mathcal{C}_{\mathcal{S B D}}\left(\mathcal{A}_{\mathcal{D I R}}\right)\right)$. Since $P$ is an idempotent in $Z\left[\mathcal{C}_{\mathcal{S B D}}\left(\mathcal{A}_{\mathcal{D I R}}\right)\right]$ and $M_{A, B}(I-P) \mathcal{C}_{\mathcal{S B D}}\left(\mathcal{A}_{\mathcal{D I R}}\right) \subseteq \operatorname{Rad}\left(\mathcal{C}_{\mathcal{S B D}}\left(\mathcal{A}_{\mathcal{D I R}}\right)\right)$ then $M_{A, B}$ is a homomorphism with $A X Y B+\operatorname{Rad}\left(\mathcal{C}_{\mathcal{S B D}}\left(\mathcal{A}_{\mathcal{D I R}}\right)\right)=A X P Y B+\operatorname{Rad}\left(\mathcal{C}_{\mathcal{S B D}}\left(\mathcal{A}_{\mathcal{D I R}}\right)\right)+A X(I-P) Y B+$ $\operatorname{Rad}\left(\mathcal{C}_{\mathcal{S B D}}\left(\mathcal{A}_{\mathcal{D I R}}\right)\right)=A X B A Y B+\operatorname{Rad}\left(\mathcal{C}_{\mathcal{S B D}}\left(\mathcal{A}_{\mathcal{D I R}}\right)\right)$ thus $P=B A$ is the central idempotent in $\mathcal{C}_{\mathcal{S B D}}\left(\mathcal{A}_{\mathcal{D I R}}\right)$ which has direct sum decomposition of two-sided multiplication elementary operator of the form, $M_{A, B}=M_{A_{1}, B_{1}}+M_{A_{2}, B_{2}}$, where $M_{A_{1}, B_{1}}$ is a homomorphism on $\mathcal{C}_{\mathcal{S B D}}\left(\mathcal{A}_{\mathcal{D I R}}\right)$ and $M_{A_{2}, B_{2}}\left(P \mathcal{C}_{\mathcal{S B D}}\left(\mathcal{A}_{\mathcal{D I R}}\right)\right)=0$ i.e if $A_{1}=P A, B_{1}=P B, A_{2}=(I-P) A$ and $B_{2}=(I-P) B$.

Proposition 3.3. Let $S_{1}, \ldots, S_{n} \in \mathcal{C}_{\mathcal{S B D}}\left(\mathcal{A}_{\mathcal{D I R}}\right)$ such that for every $i,\left(S_{i} A\right)^{2} \in \operatorname{Rad}\left(\mathcal{C}_{\mathcal{S B D}}\left(\mathcal{A}_{\mathcal{D I R}}\right)\right)$ and $\left(S_{i} B\right)\left(S_{k} A\right) \in \operatorname{Rad}\left(\mathcal{C}_{\mathcal{S B D}}\left(\mathcal{A}_{\mathcal{D I R}}\right)\right), \forall i \geq k, \forall A, B \in \mathcal{C}_{\mathcal{S B D}}\left(\mathcal{A}_{\mathcal{D I R}}\right)$. Then $S=\sum_{i=1}^{n} \lambda_{i} S_{i}$ is spectrally bounded for all $\lambda_{1}, \ldots, \lambda_{n} \in \mathbb{C}$.

Proof. Let $1 \leq m \leq q$ such that there exists $S_{i_{m}}$ which is a linear mapping, then for every $q+1 \leq m \leq n,\left(S_{i_{m}} A\right)^{2} \in \operatorname{Rad}\left(\mathcal{C}_{\mathcal{S B D}}\left(\mathcal{A}_{\mathcal{D I R}}\right)\right)$. We assume that for $\lambda_{i_{m}}=1$ for $q+1 \leq m \leq n$ and $\lambda_{1}, . ., \lambda_{n} \in \mathbb{C}$ then the proposition hold. Then fix $\lambda_{m}=\lambda_{m_{i}}$ for $1 \leq m \leq q$ and $A \in$ $\mathcal{C}_{\mathcal{S B D}}\left(\mathcal{A}_{\mathcal{D I R}}\right)$. Again, choose a non-zero complex number $\beta \notin \bigcup_{m=1}^{q} \sigma\left(\lambda_{m} A\right)$. So, if $1 \leq m \leq q$ 
then $B_{m}=\frac{\lambda_{m}}{\beta} A\left(\frac{\lambda_{m}}{\beta} A-I\right)^{-1}$. Thus

$$
\frac{\lambda_{m}}{\beta} A+B_{m}-B_{q} \frac{\lambda_{m}}{\beta}=0
$$

and

$$
\frac{\lambda_{m}}{\beta} S_{i_{m}} A+S_{i_{m}} B_{m}-\left(S_{i_{m}} B_{m}\right) \frac{\lambda_{m}}{\beta} S_{i_{m}} A=0 .
$$

This can be expressed as

$$
\left(1-\sum_{m=1}^{q} S_{i_{m}} B_{m}\right)\left(1-\sum_{m=1}^{q} \frac{\lambda_{m}}{\beta} S_{i_{m}} A-\sum_{m=q+1}^{n} \frac{1}{\beta} S_{i_{m}} A\right)=1+T,
$$

where $T=\sum_{m, r=1, m \neq r}^{q} \frac{\lambda_{r}}{\beta}\left(S_{i_{m}} B_{m}\right)\left(S_{i_{m}} A\right)-\sum_{m=q+1}^{n} \frac{1}{\beta} S_{i_{m}} A+$ $\sum_{m=1}^{q} \sum_{r=q+1}^{n} \frac{1}{\beta}\left(S_{i_{m}} B_{m}\right)\left(S_{i_{r}} A\right)$. Since $T \in \operatorname{Rad}\left(\mathcal{C}_{\mathcal{S B D}}\left(\mathcal{A}_{\mathcal{D I R}}\right)\right)$ then $1+T$ is invertible and $\beta-$ $\left(\sum_{m=1}^{q} \lambda_{m} S_{i_{m}}+\sum_{m=q+1}^{n} S_{i_{m}}\right) A$ is left invertible. Also, the boundary spectrum of $S A$ is contained in the left approximate point spectrum. It follows that $\beta \notin \delta \sigma(S A) \subseteq \bigcup_{m=1}^{q} \sigma\left(\lambda_{m} A\right)$. Thus $r(S A) \leq \max _{i \leq i \leq n}\left\{\left|\lambda_{i}\right|\right\} r(A)$, for every $A \in \mathcal{C}_{\mathcal{S B D}}\left(\mathcal{A}_{\mathcal{D I R}}\right)$.

Theorem 3.1. Suppose that $T_{A_{i}, B_{i}}=\sum_{i=1}^{n} A_{i} X B_{i} \in \mathcal{C}_{\mathcal{S B D}}\left(\mathcal{A}_{\mathcal{D I R}}\right)$ is spectrally bounded, then for every $i>j, B_{i} A_{j} \in \operatorname{Rad}\left(\mathcal{C}_{\mathcal{S B D}}\left(\mathcal{A}_{\mathcal{D I R}}\right)\right)$ and $B_{i} A_{i} \in Z\left[\mathcal{C}_{\mathcal{S B D}}\left(\mathcal{A}_{\mathcal{D I R}}\right)\right]$.

Proof. Let $\Phi: \mathcal{C}_{\mathcal{S B D}}\left(\mathcal{A}_{\mathcal{D I R}}\right) \rightarrow \Omega$ be an irreducible representation of $\mathcal{C}_{\mathcal{S B D}}\left(\mathcal{A}_{\mathcal{D I R}}\right)$ on Banach space $\Omega$ such that $T_{A_{\phi i}, B_{\phi i}}^{\phi} \in \Phi\left(\mathcal{C}_{\mathcal{S B D}}\left(\mathcal{A}_{\mathcal{D I R}}\right)\right)$ is given by $T_{A_{\phi i}, B_{\phi i}}^{\phi}=\sum_{i=1}^{n} A_{\phi i} X_{\phi} B_{\phi i}$. By Proposition 3.2, $\left(B_{\phi i}\right)\left(A_{\phi i}\right)=E_{i} I \in \mathbb{C}_{I}$ and hence the elementary operator $T_{A_{\phi i}, B_{\phi i}}^{\phi}$ is spectrally bounded with the spectral operator norm $\left\|T_{A_{i}, B_{i}}\right\|_{\sigma} \leq\left\|T_{A_{\phi i}, B_{\phi i}}^{\phi}\right\|_{\sigma}=\left\|A_{\phi i} X_{\phi i} B_{\phi i}\right\|_{\sigma} \leq\left|E_{i}\right|$. Suppose that $E_{i}=0$ then $\left(T_{A_{\phi i}, B_{\phi i}}^{\phi} \phi(X)\right)^{2}=0$ for every $X \in \mathcal{C}_{\mathcal{S B D}}\left(\mathcal{A}_{\mathcal{D I R}}\right)$. However, $E_{i}^{-1}\left(T_{A_{\phi i}, B_{\phi i}}^{\phi}\right)$ is a linear mapping. Fix $S_{i}=T_{A_{\phi i}, B_{\phi i}}^{\phi}=\sum_{i=1}^{n} A_{\phi i} X_{\phi} B_{\phi i}, 1 \leq i \leq n$ such that $B_{\phi i} A_{\phi j}=0 i>j$ then we have $S_{i} \Phi(Y) S_{j} \Phi(X)=0$, for every $X, Y \in \mathcal{C}_{\mathcal{S B D}}\left(\mathcal{A}_{\mathcal{D I R}}\right)$ and $i>j$. Using Proposition 3.3, we obtain $S_{\Phi i}=\sum_{i=1}^{n} \lambda_{i} S_{i}$ is spectrally bounded with $\left\|S_{\Phi}\right\|_{\sigma} \leq \max _{1 \leq i \leq n}\left|\lambda_{i}\right|$ where $\lambda=1$ if $E_{i}=0$ and $\lambda_{i}=E_{i}$ otherwise. Choose $\beta=1+\max _{1 \leq i \leq n}\left\|B_{i} A_{i}\right\|$ then for all $X \in \mathcal{C}_{\mathcal{S B D}}\left(\mathcal{A}_{\mathcal{D I R}}\right)$, $r(S x)=\sup _{\Phi} r\left\{T_{A_{\phi i}, B_{\phi i}}^{\phi}(X)\right\} \leq \beta r(X)$ hence $T_{A_{i}, B_{i}}$ is spectrally bounded.

Theorem 3.2. Let $\mathcal{C}_{\mathcal{S B D}}\left(\mathcal{A}_{\mathcal{D I R}}\right)$ be a unital irreducible $C^{*}$-subalgebra. If $T_{A_{i}, B_{i}} \in \mathcal{C}_{\mathcal{S B D}}\left(\mathcal{A}_{\mathcal{D I R}}\right)$ is spectrally infinitesimal i.e $r\left(T_{A_{i}, B_{i}}(X)\right)=0$, for every $X \in \mathcal{C}_{\mathcal{S B D}}\left(\mathcal{A}_{\mathcal{D I R}}\right)$ then $B_{i} A_{i} \in \operatorname{Rad}\left(\mathcal{C}_{\mathcal{S B D}}\left(\mathcal{A}_{\mathcal{D I R}}\right)\right)$.

Proof. Let $\Phi: \mathcal{C}_{\mathcal{S B D}}\left(\mathcal{A}_{\mathcal{D I R}}\right) \rightarrow \Omega$ be an irreducible representation of $\mathcal{C}_{\mathcal{S B D}}\left(\mathcal{A}_{\mathcal{D I R}}\right)$ on Banach space $\Omega$ then $r\left(T_{A_{\phi i}, B_{\phi i}}^{\phi}\left(X_{\phi}\right)\right)=0$, for every $X \in \mathcal{C}_{\mathcal{S B D}}\left(\mathcal{A}_{\mathcal{D I R}}\right)$. Using properties defined in [21] Corollary 5, we have $\Phi(X) \mathbf{C}\left(T_{A_{\phi i}, B_{\phi i}}^{\phi}\right) v \subseteq \mathbb{C} v$ and $T_{A_{\phi i}, B_{\phi i}}^{\phi} \mathbf{A}\left(T_{A_{\phi i}, B_{\phi i}}^{\phi}\right) v \subseteq \mathbf{A}\left(T_{A_{\phi i}, B_{\phi i}}^{\phi}\right) v$, for every 
$v \in \Omega_{\phi}$ and $X \in \mathcal{C}_{\mathcal{S B D}}\left(\mathcal{A}_{\mathcal{D I R}}\right)$. Thus $T_{A_{\phi i}, B_{\phi i}}^{\phi} \mid \mathbf{L}\left(T_{A_{\phi i}, B_{\phi i}}^{\phi}\right) v$ is nilpotent and $\Phi\left(\sum_{i=1}^{n} B_{i} A_{i}\right)=0$. Thus $r\left(T_{A_{i}, B_{i}}(X)\right)=0$.

At this juncture, we give results on the closure conditions for the centre of spectrally bounded compact elementary operators on dense irreducible subalgebras.

Proposition 3.4. Let $\mathcal{C}_{\mathcal{S B D}}\left(\mathcal{A}_{\mathcal{D I R}}\right)$ be a unital irreducible $C^{*}$-subalgebra and $W\left[\mathcal{C}_{\mathcal{S B D}}(\Omega)\right]$ be the quasicentre of $\mathcal{C}_{\mathcal{S B D}}\left(\mathcal{A}_{\mathcal{D I R}}\right)$. If $r\left(\delta_{A} \delta_{X}-\delta_{X} \delta_{A}\right)=0$, for every $A \in W\left[\mathcal{C}_{\mathcal{S B D}}(\Omega)\right]$ and $X \in \mathcal{C}_{\mathcal{S B D}}\left(\mathcal{A}_{\mathcal{D I R}}\right)$, then $W\left[\mathcal{C}_{\mathcal{S B D}}(\Omega)\right]$ is closed under either addition or multiplication.

Proof. Let $W\left[\mathcal{C}_{\mathcal{S B D}}(\Omega)\right]$ be closed under multiplication such that for any $A, B \in W\left[\mathcal{C}_{\mathcal{S B D}}(\Omega)\right]$ and $\lambda \in \mathbb{C}$ then $(I+\lambda A)(I+\lambda B) \in W\left[\mathcal{C}_{\mathcal{S B D}}(\Omega)\right]$. So,

$$
\begin{aligned}
(I+\lambda A)(I+\lambda B) & =I(I+\lambda B)+\lambda A(I+\lambda B) \\
& =I+\lambda B+\lambda A+\lambda^{2} A B \\
& =I+\lambda(A+B+\lambda A B),
\end{aligned}
$$

Thus $(A+B+\lambda A B) \in W\left[\mathcal{C}_{\mathcal{S B D}}(\Omega)\right]$, for every $\lambda \neq 0$. From [19] Theorem 4.3, we have $r\left(\delta_{A+B+\lambda A B}\right)=0$ for every $\lambda \neq 0$ since the function $\lambda \rightarrow r\left(\delta_{A+B+\lambda A B}\right)$ is subharmonic on $\mathbb{C}$ and vanishes at $\lambda=0$ by Vasentini's Theorem. In addition,

$$
r\left(\delta_{A+B}\right)=0, \forall A, B \in W\left[\mathcal{C}_{\mathcal{S B D}}(\Omega)\right]
$$

hence, from Equation 3.2, $W\left[\mathcal{C}_{\mathcal{S B D}}(\Omega)\right]$ is closed under addition. For instance, for $A \in W\left[\mathcal{C}_{\mathcal{S B D}}(\Omega)\right]$, $X \in \mathcal{C}_{\mathcal{S B D}}\left(\mathcal{A}_{\mathcal{D I R}}\right)$ and $\lambda \in \mathbb{C}$ then an element in $W\left[\mathcal{C}_{\mathcal{S B D}}(\Omega)\right]$ can be represented by $e^{\lambda X} A e^{-\lambda X}$ $A=\lambda \delta_{X} A+\frac{\lambda^{2}}{2 !} \delta_{X}^{2} A+\ldots=\lambda \Psi(\lambda)$. Hence, $e^{\lambda X} A e^{-\lambda X} \in W\left[\mathcal{C}_{\mathcal{S B D}}(\Omega)\right]$ and applying Equation 3.2, $r\left(\delta_{\Psi(\lambda)}\right)=0$, for every $\lambda \neq 0$ which implies that $r\left(\delta_{\Psi(0)}\right)=r\left(\delta_{A} \delta_{X}-\delta_{X} \delta_{A}\right)=0$.

Lemma 3.1. Let $X$ be in $\mathcal{C}_{\mathcal{S B D}}\left(\mathcal{A}_{\mathcal{D I R}}\right)$ such that $r\left(\delta_{A}\right)=0$ and $r\left(\delta_{A} \delta_{X}-\delta_{X} \delta_{A}\right)=0$, for every $X \in \mathcal{C}_{\mathcal{S B D}}\left(\mathcal{A}_{\mathcal{D I R}}\right)$. Then $A X-X A \in \operatorname{Rad}\left(\mathcal{C}_{\mathcal{S B D}}\left(\mathcal{A}_{\mathcal{D I R}}\right)\right)$, for every $X \in \mathcal{C}_{\mathcal{S B D}}\left(\mathcal{A}_{\mathcal{D I R}}\right)$. Moreover, $0 \in Z\left[\mathcal{C}_{\mathcal{S B D}}\left(\mathcal{A}_{\mathcal{D I R}}\right)\right]$.

Proof. Let $\mathcal{A}_{\mathcal{S B D}}$ be an irreducible $C^{*}$-subalgebra of $\mathcal{C}_{\mathcal{S B D}}\left(\mathcal{A}_{\mathcal{D I R}}\right)$ and $\mathcal{J}=\left\{X \in \mathcal{C}_{\mathcal{S B D}}\left(\mathcal{A}_{\mathcal{D I R}}\right) ; X \mathcal{A}_{\mathcal{S B D}}=\right.$ $0\}$ is the primitive ideal of $\mathcal{C}_{\mathcal{S B D}}\left(\mathcal{A}_{\mathcal{D I R}}\right)$.

(i). Let assume that for each $V \in \mathcal{A}_{\mathcal{S B D}}$ then there exists $V, A V$ and $A^{2} V$ which are linearly independent. By Jacobson Transitivity theorem there exists $X \in \mathcal{C}_{\mathcal{S B D}}\left(\mathcal{A}_{\mathcal{D I R}}\right)$ such that 
$X V=X A^{2} V=0$ and $X A V=V$. Denote the $Z=A X-X A$. In this case, we see that $Z V=-V$ and $Z A V=A V$. Now for $n=0,1,2, \ldots$ we have

$$
\left(\delta_{Z}^{n} A\right) V=2^{n} A V
$$

when $n=0$, it is trivial. Suppose that Equation 3.3 holds for $n \geq 0$, then $\left(\delta_{Z}^{n+1} A\right)=$ $\left[Z\left(\delta_{Z}^{n} A\right)-\left(\delta_{Z}^{n} A\right) Z\right] V=(Z+1) 2^{n} A V=2^{n+1} A V$ and hence $2\|A V\|^{\frac{1}{n}} \leq\left\|\delta_{Z}^{n}\right\|^{\frac{1}{n}}\|A\|^{\frac{1}{n}}\|V\|^{\frac{1}{n}}$. When $n \rightarrow \infty$, then $r\left(\delta_{Z}\right) \geq 2$ which implies $r\left(\delta_{Z}\right)=r\left(\delta_{A} \delta_{X}-\delta_{X} \delta_{A}\right) \geq 2$ is a contradiction from the first hypothesis that $r\left(\delta_{Z}\right)=0$.

(ii). From above, let $V, A V$ and $A^{2} V$ be linearly dependent for every $V \in \mathcal{A}_{\mathcal{S B D}}$ and applying [22] Theorem 4.2.7, then the action of $A$ on $\mathcal{A}_{\mathcal{S B D}}$ is algebraic degree of at most 2. suppose that $\Psi$ is the minimal polynomial of $A$ modulo $\mathcal{J}$.

(iii). Let $\rho \neq \gamma$ such that the polynomial $\Psi(\lambda)=(\lambda-\rho)(\lambda-\gamma)$ then for each element $X \in \mathcal{C}_{\mathcal{S B D}}\left(\mathcal{A}_{\mathcal{D I R}}\right)$, we have

$$
\begin{aligned}
\left(\lambda-\gamma-\delta_{A}\right)(\gamma-A) X(\rho-A) & =\left[\left(\rho-L_{A}\right)-\left(\gamma-R_{A}\right)\right](\gamma-A) X(\rho-A) \\
& =\Psi(A) X(\rho-A)-(\gamma-A) X \Psi(A)
\end{aligned}
$$

is in $\mathcal{J}$. So, $r\left(\delta_{A}\right)=0$ and for every $\lambda \neq 0,\left(\lambda-\delta_{A}\right)^{-1}=\lambda^{-1}+\lambda^{-2} \delta_{A}+\ldots$ which maps $\mathcal{J}$ into $\mathcal{J}$. Hence, $(\gamma-A) \mathcal{C}_{\mathcal{S B D}}\left(\mathcal{A}_{\mathcal{D I R}}\right)(\rho-A) \subseteq \mathcal{J}$ and either $(\rho-A) \in \mathcal{J}$ or $(\gamma-A)$ are prime which contradicts the minimality of $\Psi$.

(iv). Let $\Psi(\lambda)=(\lambda-\rho)^{2}$ be constants such that $B=A-\rho$ and $B^{2} \mathcal{A}_{\mathcal{S B D}}=0$. It follows that for each $V \in \mathcal{A}_{\mathcal{S B D}}$ then there exists $V, A V$ and $A^{2} V$ are linearly independent. So applying Jacobson Transitivity theorem, choose $Y \in \mathcal{C}_{\mathcal{S B D}}\left(\mathcal{A}_{\mathcal{D I R}}\right)$ such that $Y V=0$ and $Y B V=V$. This means that $Y B^{2} V=0$ since $\delta_{B}=\delta_{A}$. Then, we denote the $R=B Y-Y B$. In this case, we see that $R V=-V$ and $R B V=B V$. Now for $n=0,1,2, \ldots$ we have

$$
\left(\delta_{R}^{n} B\right) V=2^{n} B V
$$

when $n=0, B V=B V$ which is trivial. Suppose that Equation 3.4 holds for $n \geq 0$, then $\left(\delta_{R}^{n+1} B\right)=\left[R\left(\delta_{R}^{n} B\right)-\left(\delta_{R}^{n} B\right) R\right] V=(R+1) 2^{n} B V=2^{n+1} B V$ and hence $2\|B V\|^{\frac{1}{n}} \leq$ $\left\|\delta_{R}^{n}\right\|^{\frac{1}{n}}\|B\|^{\frac{1}{n}}\|V\|^{\frac{1}{n}}$. When $n \rightarrow \infty, r\left(\delta_{R}\right) \geq 2$ which implies $r\left(\delta_{R}\right)=r\left(\delta_{B} \delta_{Y}-\delta_{Y} \delta_{B}\right) \geq 2$ is a contradiction from the first hypothesis that $r\left(\delta_{R}\right)=0$. 
(v). Thus the only option is to choose $\rho \in \mathbb{C}$ such that $\Psi$ has a degree 1 and $A-\rho \in \mathcal{J}$. Hence $A X-X A \in \mathcal{J}$ for every $X \in \mathcal{C}_{\mathcal{S B D}}\left(\mathcal{A}_{\mathcal{D I R}}\right)$ since $\mathcal{J}$ is an arbitrary primitive ideal of $\mathcal{C}_{\mathcal{S B D}}\left(\mathcal{A}_{\mathcal{D I R}}\right)$ and $A$ is central modulo $\operatorname{Rad}\left(\mathcal{C}_{\mathcal{S B D}}\left(\mathcal{A}_{\mathcal{D I R}}\right)\right)$.

From [21] Proposition 2, we see that $Z\left[\mathcal{C}_{\mathcal{S B D}}\left(\mathcal{A}_{\mathcal{D I R}}\right)\right]$ is a commutative irreducible $C^{*}$-subalgebra. This implies that $\delta_{A} \delta_{X}=\delta_{X} \delta_{A}$ and $r\left(\delta_{A}\right)=0 \Rightarrow A=0$. Hence, $0 \in Z\left[\mathcal{C}_{\mathcal{S B D}}\left(\mathcal{A}_{\mathcal{D I R}}\right)\right]$.

Theorem 3.3. Let $\mathcal{C}_{\mathcal{S B D}}\left(\mathcal{A}_{\mathcal{D I R}}\right)$ be a semi-prime and unital irreducible $C^{*}$-subalgebra. If $Z\left[\mathcal{C}_{\mathcal{S B D}}\left(\mathcal{A}_{\mathcal{D I R}}\right)\right]$ is the centre of $\mathcal{C}_{\mathcal{S B D}}\left(\mathcal{A}_{\mathcal{D I R}}\right)$ and $W\left[\mathcal{C}_{\mathcal{S B D}}(\Omega)\right]$ is the quasicentre of $\mathcal{C}_{\mathcal{S B D}}\left(\mathcal{A}_{\mathcal{D I R}}\right)$, then the following statements are equivalent:

(i). $W\left[\mathcal{C}_{\mathcal{S B D}}(\Omega)\right]=Z\left[\mathcal{C}_{\mathcal{S B D}}\left(\mathcal{A}_{\mathcal{D I R}}\right)\right] ;$

(ii). $W\left[\mathcal{C}_{\mathcal{S B D}}(\Omega)\right]$ is closed under addition;

(iii). $W\left[\mathcal{C}_{\mathcal{S B D}}(\Omega)\right]$ is closed under multiplication;

(iv). for every $A, B \in W\left[\mathcal{C}_{\mathcal{S B D}}(\Omega)\right], A B=B A$;

(v). for every $A \in W\left[\mathcal{C}_{\mathcal{S B D}}(\Omega)\right]$ and $B \in \mathcal{C}_{\mathcal{S B D}}\left(\mathcal{A}_{\mathcal{D I R}}\right)^{-1}$ then $A$ commutes with $B^{-1} A B$;

(vi). for every $A \in W\left[\mathcal{C}_{\mathcal{S B D}}(\Omega)\right]$ and $B \in \mathcal{C}_{\mathcal{S B D}}\left(\mathcal{A}_{\mathcal{D I R}}\right)^{-1}$ then $A$ commutes with $e^{B} A e^{-B}$.

Proof. We prove that $W\left[\mathcal{C}_{\mathcal{S B D}}(\Omega)\right]=Z\left[\mathcal{C}_{\mathcal{S B D}}\left(\mathcal{A}_{\mathcal{D I R}}\right)\right]$. From Definition 2.3, the quasi-centre $W\left[\mathcal{C}_{\mathcal{S B D}}(\Omega)\right]$ of $\mathcal{C}_{\mathcal{S B D}}\left(\mathcal{A}_{\mathcal{D I R}}\right)$ is the set of all quasi-central elements $A \in \mathcal{C}_{\mathcal{S B D}}\left(\mathcal{A}_{\mathcal{D I R}}\right)$ defined by $W\left[\mathcal{C}_{\mathcal{S B D}}(\Omega)\right]=\bigcup_{\omega \geq 1}\left\{\|X(\lambda-A)\| \leq \omega\|(\lambda-A) X\|\right.$, for every $X \in \mathcal{C}_{\mathcal{S B D}}\left(A_{D I R}\right)$ and $\left.\omega \in \mathbb{K}\right\}$. If $\omega=1$ then $\|X(\lambda-A)\| \leq\|(\lambda-A) X\|$ and it follows from Definition 2.2 that $W\left[\mathcal{C}_{\mathcal{S B D}}(\Omega)\right]=$ $Z\left[\mathcal{C}_{\mathcal{S B D}}\left(\mathcal{A}_{\mathcal{D I R}}\right)\right]$. Thus $(i) \Rightarrow(i i),($ iii $)$ and $(i v)$ is trivial.

$($ ii $) \Rightarrow(\mathrm{v})$ and $($ iii $) \Rightarrow(\mathrm{v})$. Suppose that $A \in W\left[\mathcal{C}_{\mathcal{S B D}}(\Omega)\right]$ and $B \in \mathcal{C}_{\mathcal{S B D}}\left(\mathcal{A}_{\mathcal{D I R}}\right)^{-1}$ then from Proposition 3.4, either (ii) or (iii) holds and by Lemma 3.1, we have $A B-B A \in$ $\operatorname{Rad}\left(\mathcal{C}_{\mathcal{S B D}}\left(\mathcal{A}_{\mathcal{D I R}}\right)\right)$ and thus,

$$
B^{-1} A B-A=B^{-1} A B-\left(B^{-1} B\right) A=B^{-1}(A B-B A) \in \operatorname{Rad}\left(\mathcal{C}_{\mathcal{S B D}}\left(\mathcal{A}_{\mathcal{D I R}}\right)\right) .
$$

In addition, from Proposition 3.4, (ii) $W\left[\mathcal{C}_{\mathcal{S B D}}(\Omega)\right]$ is closed under addition holds hence

$$
B^{-1} A B-A \in W\left[\mathcal{C}_{\mathcal{S B D}}(\Omega)\right]
$$

similarly, from Proposition 3.4, (iii) $\Rightarrow\left(\mathrm{v}\right.$ ) holds since $B^{-1} A B-A \in W\left[\mathcal{C}_{\mathcal{S B D}}(\Omega)\right]$. In particular, let $A \in W\left[\mathcal{C}_{\mathcal{S B D}}(\Omega)\right]$ and $B \in \mathcal{C}_{\mathcal{S B D}}\left(\mathcal{A}_{\mathcal{D I R}}\right)^{-1}$ then $A$ commutes with $B^{-1} A B$ since if we substitute $A$ with a scalar $\rho$ such that $A$ is invertible then $B^{-1} A B-A=B^{-1} A B(I-$ $B^{-1} A^{-1} B A$ ). Thus Equation 3.6 and Proposition 3.4 shows that (iii) holds. Furthermore, $W\left[\mathcal{C}_{\mathcal{S B D}}(\Omega)\right] \cap \operatorname{Rad}\left(\mathcal{C}_{\mathcal{S B D}}\left(\mathcal{A}_{\mathcal{D I R}}\right)\right) \subseteq Z\left[\mathcal{C}_{\mathcal{S B D}}\left(\mathcal{A}_{\mathcal{D I R}}\right)\right]$ and by Equation 3.5 and Equation 3.6, we 
have $A$ commuting with $B^{-1} A B$.

(vi) $\Rightarrow$ (i). Let $A \in W\left[\mathcal{C}_{\mathcal{S B D}}(\Omega)\right.$ and $B \in \mathcal{C}_{\mathcal{S B D}}\left(\mathcal{A}_{\mathcal{D I R}}\right)^{-1}$. It follows that for every $\lambda \in \mathbb{C}$, then (vi) shows that $A$ commutes with $e^{\lambda X} A e^{-\lambda X}$. Fix the coefficients of $\lambda$ on both sides of the equation $A(I+\lambda X+\ldots) A(I-\lambda X+\ldots)=(I+\lambda X+\ldots) A(I-\lambda X+\ldots) A$, which gives $A X A-A^{2} X=X A^{2}-A X A$ therefore $\delta_{A}^{2} X=0$, for every $X \in \mathcal{C}_{\mathcal{S B D}}\left(\mathcal{A}_{\mathcal{D I R}}\right)$. Hence, $\delta_{A}=0$ and $A \in Z\left[\mathcal{C}_{\mathcal{S B D}}\left(\mathcal{A}_{\mathcal{D I R}}\right)\right]$. Since (vi). $\Rightarrow(\mathrm{i})$. and whenever $\omega=1$ then $W\left[\mathcal{C}_{\mathcal{S B D}}(\Omega)\right]=$ $Z\left[\mathcal{C}_{\mathcal{S B D}}\left(\mathcal{A}_{\mathcal{D I R}}\right)\right]$ hold. Thus (i) $\Rightarrow$ (ii) and it follows from Proposition 3.4, that (ii) and (iii) $\Rightarrow$ (iv) and vice-versa. Consequently (iv) $\Rightarrow$ (i) since the centre and quasi-centre are commutative.

Theorem 3.4. Let $A \in W\left[\mathcal{C}_{\mathcal{S B D}}(\Omega)\right]$ and $B \in \mathcal{C}_{\mathcal{S B D}}\left(\mathcal{A}_{\mathcal{D I R}}\right)$. Suppose that $A B$ and $B A$ are linearly dependent such that $A B=B A$ then $A B \in W\left[\mathcal{C}_{\mathcal{S B D}}(\Omega)\right] \cap \overline{Z\left[\mathcal{C}_{\mathcal{S B D}}\left(\mathcal{A}_{\mathcal{D I R}}\right)\right]}$.

Proof. Let $\rho, \gamma \in \mathbb{C}$ such that $\rho A B+\gamma B A=0$ and $\rho+\gamma \neq 0$. It follows that $\left(\rho L_{A}+\gamma R_{A}\right) B=$ $\left[(\rho+\gamma) L_{A}-\gamma \delta_{A}\right] B=0$ and $A B=L_{A} B=\kappa \delta_{A} B$ for some $\kappa \in \mathbb{C}$. We know that $L_{A}$ and $\delta_{A}$ commute, hence $A^{n} B=\kappa^{n} \delta_{A}^{n} B$ for $n=1,2, \ldots$ and by Lemma 3.1 (iii), $\left\|A^{n} B\right\|^{\frac{1}{n}} \rightarrow 0$ as $n \rightarrow \infty$. Thus for any $\lambda \neq 0 \in \mathbb{C}$ then the series $\Psi(\lambda)=\lambda^{-1} B+\lambda^{-2} A B+\lambda^{-3} A^{2} B+\ldots$ converges in $\mathcal{C}_{\mathcal{S B D}}\left(\mathcal{A}_{\mathcal{D I R}}\right)$ and $(\lambda-A) \Psi(\lambda)=B$. Suppose that $A \in W\left[\mathcal{C}_{\mathcal{S B D}}(\Omega)\right]$ then $\|\Psi(\lambda)(\lambda-A)\| \leq$ $\omega\|(\lambda-A) \Psi(\lambda)\|=\omega\|B\|$, for every $\lambda \neq 0 \in \mathbb{C}$. Applying Liouville's Theorem, $\Psi(\lambda)(\lambda-A)=$ $B+\lambda^{-1}(A B-B A)+\ldots$ is constant and $A B=B A$. Thus the hypothesis $\rho+\gamma \neq 0$ contradiction. From Theorem 3.3, $A B=B A$ is commutative and $W\left[\mathcal{C}_{\mathcal{S B D}}(\Omega)\right]=Z\left[\mathcal{C}_{\mathcal{S B D}}\left(\mathcal{A}_{\mathcal{D I R}}\right)\right]$ which

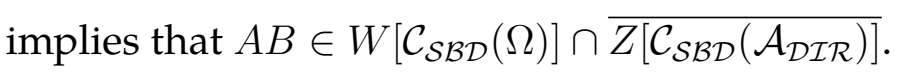

Example 3.1. Let $\left[\mathcal{C}_{\mathcal{S B D}}\left(\mathcal{A}_{\mathcal{D I R}}\right)\right]$ be a unital irreducible $C^{*}$-subalgebra with a closed quasi-centre $W\left[\mathcal{C}_{\mathcal{S B D}}(\Omega)\right]$. Then there exists a commutative sub-algebra of $\left[\mathcal{C}_{\mathcal{S B D}}\left(\mathcal{A}_{\mathcal{D I R}}\right)\right]$ that is strictly larger than its centre $Z\left[\mathcal{C}_{\mathcal{S B D}}\left(\mathcal{A}_{\mathcal{D I R}}\right)\right]$.

Proof. Let $D=\{\alpha \in \mathbb{C}:|\alpha| \leq 1\}$ be a unit disk and $\mathcal{A}_{\mathcal{S B D}}(D)$ be uniform algebra of all continuous functions $D^{n}$ and analytic in its interior $n=1,2$. Let $X \in\left[\mathcal{C}_{\mathcal{S B D}}\left(\mathcal{A}_{\mathcal{D I R}}\right)\right]$ and $\rho, \gamma \in \mathbb{C}$ such that

$$
X=\varphi+\Phi M+\rho N+\sum_{n=1}^{\infty} \gamma_{n} R_{n}
$$

for all $\varphi, \Phi \in \mathcal{A}_{\mathcal{S B D}}(D), M, N$ and $R_{n}$ are indeterminate. Then $\left[\mathcal{C}_{\mathcal{S B D}}\left(\mathcal{A}_{\mathcal{D I R}}\right)\right]$ is a Banach space with the norm $\|X\|=\|\varphi\|+\|\Phi\|+|\rho|+\sum_{n=1}^{\infty}\left|\gamma_{n}\right|<\infty$. Let $I$ be an identity function in $D$ such that $I M-M I=N, M R_{1}=N, R_{n} R_{n+1}=N$ for every $n=1,2, . ., M^{2}=N^{2}=R_{n}^{2}=0$ 
and the product of any ordered pair of generators not mentioned above $\left\{I, M, N, R_{n}\right\}=0$. This form of multiplication can be extended to $\mathcal{C}_{\mathcal{S B D}}\left(\mathcal{A}_{\mathcal{D I R}}\right)$, that is, for $\varphi \in \mathcal{A}_{\mathcal{S B D}}(D) ; M \varphi=$ $\varphi M-\varphi^{\prime}(0) N, N \varphi=\varphi N=\varphi(0) N, R_{n} \varphi=\varphi R_{n}=\varphi(0) R_{n}$ and we apply distributive law to obtain any product of elements of $\mathcal{C}_{\mathcal{S B D}}\left(\mathcal{A}_{\mathcal{D I R}}\right)$ and $N \in Z\left[\mathcal{C}_{\mathcal{S B D}}\left(\mathcal{A}_{\mathcal{D I R}}\right)\right]$. Since $\mathcal{C}_{\mathcal{S B D}}\left(\mathcal{A}_{\mathcal{D I R}}\right)$ is a Banach algebra then for $X_{1}, X_{2} \in \mathcal{C}_{\mathcal{S B D}}\left(\mathcal{A}_{\mathcal{D I R}}\right)$ then $\left\|X_{1} X_{2}\right\| \leq 2\left\|X_{1}\right\|\left\|X_{2}\right\|$. We need to prove the following statements:

$$
W\left[\mathcal{C}_{\mathcal{S B D}}(\Omega)\right]=\{\varphi+\Phi M+\rho N: \Phi(0)=0\}
$$

and

$$
Z\left[\mathcal{C}_{\mathcal{S B D}}\left(\mathcal{A}_{\mathcal{D I R}}\right)\right]=\left\{\varphi+\Phi M+\rho N: \Phi(0)=\varphi^{\prime}(0)=0\right\}
$$

Suppose that $X \in W\left[\mathcal{C}_{\mathcal{S B D}}(\Omega)\right]$ in Equation 3.7. We substitute $X$ with $X-\varphi(0)$, so that $\varphi(0)=0$. Thus $X R_{1}=\Phi(0) N, R_{1} X=\gamma_{2} N$ and for any $n \geq 2, X R_{n}=\gamma_{n-1} N, R_{n} X=\gamma_{n+1} N$. We see that $X$ commutes with $R_{n}$ for all $n$ and $\Phi(0)=\gamma_{2}=\gamma_{4}=\ldots, \gamma_{1}=\gamma_{2}=\ldots$ hence $\sum\left|\gamma_{n}\right|<\infty$. This shows that $\Phi(0)=\varphi_{n}=0 \forall n$. Thus $Z\left[\mathcal{C}_{\mathcal{S B D}}\left(\mathcal{A}_{\mathcal{D I R}}\right)\right] \subseteq W\left[\mathcal{C}_{\mathcal{S B D}}(\Omega)\right] \subseteq$ $\{\varphi+\Phi M+\rho N: \Phi(0)=0\}$. Conversely, the expression $X=\varphi+\Phi M+\rho N$ with $\Phi(0)=0$ commutes with $\gamma_{n} \forall n \geq 1$. That is, $(\Phi M) I-I(\Phi M)=\Phi(M I-I M)=-\Phi(0) N=0$, we see that $X$ commutes with $I$ and hence it commutes with all the generators $\left\{I, M, N, R_{n}\right\}$ of $\mathcal{C}_{\mathcal{S B D}}\left(\mathcal{A}_{\mathcal{D I R}}\right)$ except $M$. We find that $M \Phi M=\left(\Phi M-\Phi^{\prime}(0) N\right) M=0=\Phi M^{2}$ so that $M X-X M=M \varphi-\varphi M=-\varphi^{\prime}(0) N=0$ if and only if $\varphi^{\prime}(0)=0$ hence Equation 3.9 holds which gives characterization of $Z\left[\mathcal{C}_{\mathcal{S B D}}\left(\mathcal{A}_{\mathcal{D I R}}\right)\right]$.

We need to prove that any element with the expression $X_{1}=\varphi_{1}+\Phi_{1} M+\rho_{1} N: \Phi_{1}(0)=0$ is quasi-central. Suppose that $\varphi_{1}(0)=0$. Then using Equation 3.7, fix any $X \in \mathcal{C}_{\mathcal{S B D}}\left(\mathcal{A}_{\mathcal{D I R}}\right)$ and $\lambda \in \mathbb{C}$ such that $\Phi_{1} M+\rho_{1} N \in Z\left[\mathcal{C}_{\mathcal{S B D}}\left(\mathcal{A}_{\mathcal{D I R}}\right)\right]$, then $X_{1} X-X_{1} X=\varphi_{1} X-X \varphi_{1}=$ $\Phi\left(\varphi_{1} M-M \varphi_{1}\right)=\varphi^{\prime}(0) \Phi(0) N$ which gives $\left(\lambda-X_{1}\right) X=\left(\lambda-\varphi_{1}\right) \varphi+\left[\left(\lambda-\varphi_{1}\right) \Phi-\Phi_{1} \varphi\right] M+$ $\left[\lambda \rho-\rho_{1} \varphi(0)\right] N+\lambda \sum_{n=1}^{\infty} \gamma_{n} R_{n}$. We notice that there is no term involving $\varphi^{\prime}(0)$ since $\Phi_{1}(0)=$ $0:\left(\Phi_{1} M\right) \varphi=\Phi_{1}\left(\varphi M-\varphi^{\prime}(0) N\right)=\Phi_{1} \varphi M-\varphi^{\prime}(0) \Phi_{1}(0) N=\Phi_{1} \varphi M$. Thus,

$$
\left\|X_{1} X-X X_{1}\right\|=\left|\varphi_{1}^{\prime}(0) \| \Phi(0)\right|
$$

$$
\left\|\left(\lambda-X_{1}\right) X\right\| \geq\left\|\left(\lambda-\varphi_{1}\right) \varphi\right\|+\left\|\left(\lambda-\varphi_{1}\right) \Phi-\Phi_{1} \varphi\right\|
$$

If $\varphi_{1}=0$ then $X_{1} \in Z\left[\mathcal{C}_{\mathcal{S B D}}\left(\mathcal{A}_{\mathcal{D I R}}\right)\right] \subseteq W\left[\mathcal{C}_{\mathcal{S B D}}(\Omega)\right]$. Otherwise, there exists constant $\eta$ depending only on $\varphi_{1}$ such that $|\Phi(0)| \leq \eta\left\|\left(\lambda-\varphi_{1}\right)^{2} \Phi\right\| \forall \Phi \in \mathcal{A}_{\mathcal{S B D}}(D)$ and $\lambda \in \mathbb{C}$. 
Fix any $\Phi \in \mathcal{A}_{\mathcal{S B D}}(D)$ and $\lambda \in \mathbb{C}$. Assume that $\left\|\left(\lambda-\varphi_{1}\right) \Phi-\Phi_{1} \varphi\right\| \leq \frac{1}{6 \eta\left\|X_{1}\right\|}|\Phi(0)|$ and that $|\lambda| \leq 2\left\|X_{1}\right\|$. Then $\left\|\lambda-\Phi_{1}\right\| \leq 3\left\|X_{1}\right\|$ and therefore, $\left\|\left(\lambda-\varphi_{1}\right)^{2} \Phi-\left(\lambda-\varphi_{1}\right) \Phi_{1} \varphi\right\| \leq \frac{1}{2 \eta}|\Phi(0)|$. We find that

$$
|\Phi(0)| \leq 2 \eta\left\|\Phi_{1}\right\|\left\|\left(\lambda-\varphi_{1}\right) \varphi\right\| \leq 2 \eta\left\|\Phi_{1}\right\|\left\|\left(\lambda-X_{1}\right) X\right\|
$$

However, if $|\lambda| \leq 2\left\|X_{1}\right\|$, the we have

$$
|\Phi(0)| \leq 6 \eta\left\|X_{1}\right\|\left\|\left(\lambda-\varphi_{1}\right) \Phi-\Phi_{1} \varphi\right\| \leq 6 \eta\left\|X_{1}\right\|\left\|\left(\lambda-X_{1}\right) X\right\|
$$

We see that from Equation 3.10, 3.12 and 3.13, there exists a constant $\Omega$ depending only on $X_{1}$ such that $\left\|X\left(\lambda-X_{1}\right)\right\| \leq\left\|\left(\lambda-X_{1}\right) X\right\|+\left\|X_{1} X-X X_{1}\right\| \leq 6 \eta\left\|X_{1}\right\|\left\|\left(\lambda-X_{1}\right) X\right\| \forall X \in \mathcal{C}_{\mathcal{S B D}} \mathcal{E}$ and $\|\lambda\| \leq 2\left\|X_{1}\right\|$ hence $X_{1} \in W\left[\mathcal{C}_{\mathcal{S B D}}(\Omega)\right]$. This shows that Equation 3.8 holds. We also find that $W\left[\mathcal{C}_{\mathcal{S B D}}(\Omega)\right]$ is commutative $C^{*}$-subalgebra of $\mathcal{C}_{\mathcal{S B D}}\left(\mathcal{A}_{\mathcal{D I R}}\right)$. For instance, if $\Phi_{1}(0)=\Phi_{2}(0)=0$, then $\left(\varphi_{1}+\Phi_{1} M+\rho_{1} N\right)\left(\varphi_{2}+\Phi_{2} M+\rho_{2} N\right)=\varphi_{1} \varphi_{2}+\left(\varphi_{1} \Phi_{2}+\varphi_{2} \Phi_{1}\right) M+\left[\rho_{2} \varphi_{1}(0)+\rho_{1} \varphi_{2}(0)\right] N$.

Proposition 3.5. Let $Z\left[\mathcal{C}_{\mathcal{S B D}}\left(A_{D I R}\right)\right]$ be the centre of an irreducible $C^{*}$-subalgebra of $\mathcal{C}_{\mathcal{S B D}}\left(\mathcal{A}_{\mathcal{D I R}}\right)$. Then the closure of the centre $\overline{Z\left[\mathcal{C}_{\mathcal{S B D}}\left(\mathcal{A}_{\mathcal{D I R}}\right)\right]}$ is closed and is contained in any closed $C^{*}$-subalgebra $\mathcal{A}_{\mathcal{S B D}}$ of $\mathcal{C}_{\mathcal{S B D}}\left(\mathcal{A}_{\mathcal{D I R}}\right)$

Proof. Suppose that $Z\left[\mathcal{C}_{\mathcal{S B D}}\left(\mathcal{A}_{\mathcal{D I R}}\right)\right]$ is open, then the closure of the centre $\overline{Z\left[\mathcal{C}_{\mathcal{S B D}}\left(\mathcal{A}_{\mathcal{D I R}}\right)\right]}$ is equal to $\operatorname{Int}\left(\mathcal{C}_{\mathcal{S B D}}\left(\mathcal{A}_{\mathcal{D I R}}\right) \backslash Z\left[\mathcal{C}_{\mathcal{S B D}}\left(\mathcal{A}_{\mathcal{D I R}}\right)\right]\right)$ is open, thus $\left.\overline{Z\left[\mathcal{C}_{\mathcal{S B D}}\left(A_{D I R}\right)\right.}\right]$ is closed. If $\mathcal{A}_{\mathcal{S B D}}$ is closed subalgebra which contains the centre $Z\left[\mathcal{C}_{\mathcal{S B D}}\left(\mathcal{A}_{\mathcal{D I R}}\right)\right]$, we need to prove $\mathcal{A}_{\mathcal{S B D}}$ contains $\overline{Z\left[\mathcal{C}_{\mathcal{S B D}}\left(\mathcal{A}_{\mathcal{D I R}}\right)\right]}$. Then every point $X \in \overline{Z\left[\mathcal{C}_{\mathcal{S B D}}\left(\mathcal{A}_{\mathcal{D I R}}\right)\right]}$ is the limit of the sequence of elements in $Z\left[\mathcal{C}_{\mathcal{S B D}}\left(\mathcal{A}_{\mathcal{D I R}}\right)\right] \subseteq \mathcal{A}_{\mathcal{S B D}}$. Since also $\mathcal{A}_{\mathcal{S B D}}$ is closed it implies that $X \in \mathcal{A}_{\mathcal{S B D}}$ and hence

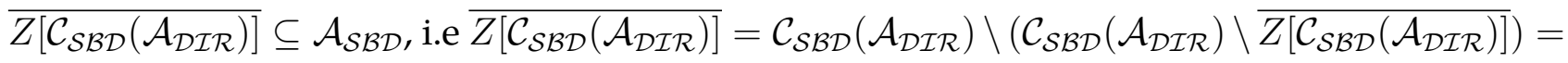
$\mathcal{C}_{\mathcal{S B D}}\left(\mathcal{A}_{\mathcal{D I R}}\right) \backslash \operatorname{Int}\left(\mathcal{C}_{\mathcal{S B D}}\left(\mathcal{A}_{\mathcal{D I R}}\right) \backslash Z\left[\mathcal{C}_{\mathcal{S B D}}\left(\mathcal{A}_{\mathcal{D I R}}\right)\right]\right) \subseteq \mathcal{C}_{\mathcal{S B D}}\left(\mathcal{A}_{\mathcal{D I R}}\right) \backslash\left(\mathcal{C}_{\mathcal{S B D}}\left(\mathcal{A}_{\mathcal{D I R}}\right) \backslash \mathcal{A}_{\mathcal{S B D}}\right) \subseteq$ $\mathcal{A}_{\mathcal{S B D}}$.

Theorem 3.5. Let $\mathcal{A}_{\mathcal{S B D}}$ be a closed $C^{*}$-subalgebra of $\mathcal{C}_{\mathcal{S B D}}\left(\mathcal{A}_{\mathcal{D I R}}\right)$ and $\mathcal{B}_{\mathcal{S B D}}$ be an open $C^{*}$-subalgebra of $\mathcal{C}_{\mathcal{S B D}}\left(\mathcal{A}_{\mathcal{D I R}}\right)$. Then the centre $Z\left[\mathcal{C}_{\mathcal{S B D}}\left(\mathcal{A}_{\mathcal{D I R}}\right)\right]$ is closed in $\mathcal{C}_{\mathcal{S B D}}\left(\mathcal{A}_{\mathcal{D I R}}\right)$ with the closure of the centre

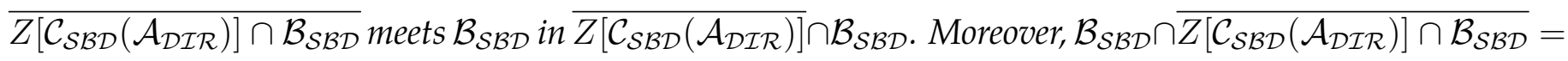
$\mathcal{A}_{\mathcal{S B D}} \cap \mathcal{B}_{\mathcal{S B D}}$

Proof. It is known that $\overline{Z\left[\mathcal{C}_{\mathcal{S B D}}\left(\mathcal{A}_{\mathcal{D I R}}\right)\right]} \cap \mathcal{B}_{\mathcal{S B D}}$ is a closed subalgebra in $\mathcal{B}_{\mathcal{S B D}}$ since it contains $Z\left[\mathcal{C}_{\mathcal{S B D}}\left(\mathcal{A}_{\mathcal{D I R}}\right)\right] \cap \mathcal{B}_{\mathcal{S B D}}$. We need to show that for every point $X \in \overline{Z\left[\mathcal{C}_{\mathcal{S B D}}\left(\mathcal{A}_{\mathcal{D I R}}\right)\right]} \cap \mathcal{B}_{\mathcal{S B D}}$ is limit point of the sequence every $X_{n} \in Z\left[\mathcal{C}_{\mathcal{S B D}}\left(\mathcal{A}_{\mathcal{D I R}}\right)\right] \cap \mathcal{B}_{\mathcal{S B D}}$. Thus, for every $X \in \overline{Z\left[\mathcal{C}_{\mathcal{S B D}}\left(\mathcal{A}_{\mathcal{D I R}}\right)\right]}$ 
then $\lim _{n \rightarrow \infty} X_{n}=X$ for every $X_{n} \in Z\left[\mathcal{C}_{\mathcal{S B D}}\left(\mathcal{A}_{\mathcal{D I R}}\right)\right]$ hold. Since $X \in \mathcal{B}_{\mathcal{S B D}}$ is open, then there exists unit disk $D=\left\{\|X\| \leq 1, \forall, X \in \mathcal{A}_{\mathcal{S B D}}\right\} \subseteq \mathcal{B}_{\mathcal{S B D}}$ and $X_{n}$ converges to $X$ with $\left\{X_{n}\right\} \in$ $Z\left[\mathcal{C}_{\mathcal{S B D}}\left(\mathcal{A}_{\mathcal{D I R}}\right)\right] \cap \mathcal{B}_{\mathcal{S B D}}$. Now, let $\mathcal{B}_{\mathcal{S B D}}$ be an interior of closed subalgebra $\mathcal{B}_{\mathcal{S B D}}$. Since $\mathcal{A}_{\mathcal{S B D}}$ is a closed subalgebra containing $\mathcal{B}_{\mathcal{S B D}}$, it follows that $\mathcal{A}_{\mathcal{S B D}}$ contains $\overline{\mathcal{B}_{\mathcal{S B D}}}$ and since $\mathcal{B}_{\mathcal{S B D}}$ is open, then $\overline{\mathcal{B}_{\mathcal{S B D}}}$ must lie in the interior of $\mathcal{B}_{\mathcal{S B D}}$ with $\mathcal{B}_{\mathcal{S B D}} \subseteq \operatorname{Int}\left(\overline{\mathcal{B}_{\mathcal{S B D}}}\right) \subseteq \operatorname{int}\left(\mathcal{A}_{\mathcal{S B D}}\right)=\mathcal{B}_{\mathcal{S B D}}$

\section{ACKNOWLEDGEMENTS}

The authors are grateful to the referees for the useful comments.

\section{Author Contribution}

All authors contributed equally to the writing of this paper and the authors declare that there is no any conflict of interest. All authors read and approved the final manuscript.

\section{REFERENCES}

[1] P. Ara, M. Mathieu, Local Multiplier of $C^{*}$-algebras, Springer-Verlag, London, 2002.

[2] M. Mathieu, Where is the Image of Derivation, Banach Centre Publ. 30 (1) (1994), 237-249.

[3] Y. F. Lin, M. Mathieu, Jordan Isomorphisms of Purely Infinite $C^{*}$-algebras, Quart. J. Math. 58 (2007), 249-253.

[4] N. B. Okelo, J. O. Agure, A Two-sided Multiplication Operator Norm, Gen. Math. Notes, 2(1) (2011), 18-23.

[5] N. B. Okelo, J. O. Agure, P. O. Oleche, Certain Conditions for Norm-attainability of Elementary Operators and Derivations, Int. J. Math. Soft Comput. 3(1) (2013), 53-59.

[6] N. B. Okelo, Various Notions of Norm-attainability in Normed Spaces, arXiv:2004.05496v1, 2013.

[7] N. B. Okelo, J. O. Agure, D. O. Ambogo, Norms of elementary operators and characterization of normattainable operators, Int. J. Math. Anal. 24 (2010), 1197-1204.

[8] M. Bresar, Y. V. Turovskii, Compactness Conditions for Elementary Operators, Stud. Math. 178(6) (2007), $1-18$.

[9] L. Molnar, P. Semrl, Elementary Operators on Standard Operators, Linear Multilinear Alg. 50(4) (2002), 315-319.

[10] N. Boudi, M. Mathieu, Elementary Operators that are Spectrally Bounded, $3^{\text {rd }}$ International Conference on Elementary Operators and their Applications, Queen's University Belfast, Belfast, 2009.

[11] M. Mathieu, A Collection of Problems on Spectrally Bounded Operators, Asian-Eur. J. Math. 2(3) (2009), 487-501.

[12] M. Young, Structure of Spectrally of Spectrally Bounded Operators on Banach Algebras, Irish Math. Soc. Bull. 77 (2016), 7-8.

[13] M. Mathieu, M. Young, Spectrally Isometric Elementary Operators, Lecture Notes, Pure Mathematics Research Centre, Queen's University Belfast, North Ireland, 2016. 
[14] J. F. Rennison, Conditions Related to Centrality in Banach Algebra II, J. Lond. Math. Soc. 2(35) (1987), 499-513.

[15] J. Sarsuor, Y. A. As'ad, Some Properties of Centrality in a Complex Banach Algebra, J. Islamic Univ. Gaza, 9(2) (2001), 1-9.

[16] Y. A. As'ad, Some Remarks of Centrality in Non-unital Complex Banach Algebra, Southeast Asian Bull. Math. 30 (2006), 1008-1015.

[17] Y. A. As'ad, Extended Centerality in Complex Banach Algebra, Int. Math. Forum, 3(3) (2018), 117-122.

[18] B. P. Duggal, On the Range Closure of an Elementary Operator, Linear Alg. Appl. 402 (2005), 199-206.

[19] J. F. Rennison, Conditions Related to Centrality in Banach Algebra, J. Lond. Math. Soc. 2(26) (1982), 155-168.

[20] Y. A. As'ad, Centrality in Banach Algebra, Masters Thesis, University of Jordan, Jordan, (1989).

[21] W. Kangogo, N. B. Okelo, O. Ongati, On Centre Properties of Irreducible Subalgebras of Compact Elementary Operators, Open J. Math. Anal. 4(2) (2020), 80-88.

[22] B. Aupetit, A Primer on Spectral Theory, Springer-verlag, New York, 1990. 